\title{
The Operator Splitting Method for Black-Scholes Equation
}

\author{
Yassir Daoud $^{1,2}$, Turgut Öziş ${ }^{2 *}$ \\ ${ }^{1}$ Faculty of Technology of Mathematical Sciences \& Statistic, El-Neelain University, Khartoum, Sudan \\ ${ }^{2}$ Department of Mathematics, Faculty of Science, Ege University, Bornova, Turkey \\ E-mail: turgut.ozis@ege.edu.tr
}

Received December 7, 2010; revised May 6, 2011; accepted May 9, 2011

\begin{abstract}
The Operator Splitting method is applied to differential equations occurring as mathematical models in financial models. This paper provides various operator splitting methods to obtain an effective and accurate solution to the Black-Scholes equation with appropriate boundary conditions for a European option pricing problem. Finally brief comparisons of option prices are given by different models.
\end{abstract}

Keywords: Operator Splitting Method, Black-Scholes Equation, European Option Pricing

\section{Introduction}

Finance is one of the most rapidly changing and fastest growing areas in the corporate business world. Because of this rapid change, modern financial instruments have become extremely complex. New mathematical models are essential to implement and price these new financial instruments. The world of corporate finance once managed by business students is now controlled by mathematicians and computer scientists. In the early 1970's, Merton [1,2] and Black and Merton [3], made an important breakthrough in the pricing of complex financial instruments by developing what has become known as the Black-Scholes model. Originally, their models are formulated in terms of stochastic differential equations. Under certain restrictive assumptions, these models are written as linear evolutionary partial differential equations with variable coefficients. The Black-Scholes model displayed the importance that mathematics plays in the field of finance. It also led to the growth and success of the new field of mathematical finance or financial engineering [4-10].

In this paper, first, we will give the derivation of the Black-Scholes partial differential equation [4] once more to refresh the minds and ultimately solve the equation for a European call option with the variants of Operator Splitting method.

\section{Derivation of the Black-Scholes Equation and Its Similarity Solution}

In this section, the price of a derivative security $V(S ; t)$ is re-derived [4]. We let the option $V(s ; t)$ whose value depends only on $S$ and $t$, and the option $V(S ; t)$ be, at least, twice differentiable in $S$ and differentiable in $t$. It is not necessary at this stage to specify whether $V(S ; t)$ is a call or a put option. In fact, $V(S ; t)$ can be the value of a whole portfolio of different options although for simplicity the reader can think of a simple call or put.

From Ito's process we have

$$
\mathrm{d} V(S, t)=\frac{\partial V}{\partial S} \mathrm{~d} S+\frac{\partial V}{\partial t} \mathrm{~d} t+\frac{1}{2} \sigma^{2} S^{2} \frac{\partial^{2} V}{\partial S^{2}} \mathrm{~d} t
$$

This gives the random walk followed by $V(S ; t)$.

Now suppose that at time $t$ the asset price is $S$ which obeys to stochastic differential equation

$$
\frac{\mathrm{d} S}{\mathrm{~S}}=\sigma \mathrm{d} X+\mu \mathrm{d} t
$$

where $\sigma$ is a number called volatility and $\mu$ is a measure of the average rate of growth of the asset price, also known as the drift.

Plugging (2) into (1) for $\mathrm{d} S$, we have

$$
\mathrm{d} V(S, t)=\frac{\partial V}{\partial S}(\mu S \mathrm{~d} t+\sigma S \mathrm{~d} B)+\frac{\partial V}{\partial t} \mathrm{~d} t+\frac{1}{2} \sigma^{2} S^{2} \frac{\partial^{2} V}{\partial S^{2}} \mathrm{~d} t
$$

and this simplifies to

$$
\mathrm{d} V(S, t)=\sigma S \mathrm{~d} B \frac{\partial V}{\partial S}+\left(\mu S \frac{\partial V}{\partial S}+\frac{\partial V}{\partial t}+\frac{1}{2} \sigma^{2} S^{2} \frac{\partial^{2} V}{\partial S^{2}}\right) \mathrm{d} t
$$

Now set up a portfolio long one option, $V$, and short an 
amount $\frac{\partial V}{\partial S}$ stock.

Note from above that this portfolio is hedged. The value of this portfolio, $\pi$, is

$$
\pi=V-\frac{\partial V}{\partial S} S
$$

The change, $d \pi$, in the value of this portfolio over a small time interval $\mathrm{d} t$ is given by

$$
\mathrm{d} \pi=\mathrm{d} V-\frac{\partial V}{\partial S} \mathrm{~d} S
$$

Now plugging (3) and stochastic differential equation into (5) for $d V$ and $d S$ we get

$$
\begin{aligned}
\mathrm{d} \pi= & \sigma S \mathrm{~d} B \frac{\partial V}{\partial S} \mathrm{~d} B+\left(\mu S \frac{\partial V}{\partial S}+\frac{\partial V}{\partial t}+\frac{1}{2} \sigma^{2} S^{2} \frac{\partial^{2} V}{\partial S^{2}}\right) \mathrm{d} t \\
& -\frac{\partial V}{\partial S}(\mu S \mathrm{~d} t+\sigma S \mathrm{~d} B)
\end{aligned}
$$

This simplifies to

$$
\mathrm{d} \pi=\left(\frac{\partial V}{\partial t}+\frac{1}{2} \sigma^{2} S^{2} \frac{\partial^{2} V}{\partial S^{2}}\right) \mathrm{d} t
$$

It is important to note that this portfolio is completely riskless because it does not contain the random Brownian motion term. Since this portfolio contains no risk it must earn the same as other short-term risk-free securities. If it earned more than this, arbitrageurs could make a profit by shorting the riskfree securities and using the proceeds to buy this portfolio. If the portfolio earned less arbitrageurs could make a riskless profit by shorting the portfolio and buying the risk-free securities. It follows for a riskless portfolio that

$$
\mathrm{d} \pi=r \pi \mathrm{d} t
$$

where $r$ is the risk free interest rate. Substituting for $\mathrm{d} \pi$ and $\pi$ from (6) and (3) yields

$$
\left(\frac{\partial V}{\partial t}+\frac{1}{2} \sigma^{2} S^{2} \frac{\partial^{2} V}{\partial S^{2}}\right) \mathrm{d} t=r\left(V-S \frac{\partial V}{\partial S}\right) \mathrm{d} t
$$

or

$$
\frac{\partial V}{\partial t}+\frac{1}{2} \sigma^{2} S^{2} \frac{\partial^{2} V}{\partial S^{2}}+r S \frac{\partial V}{\partial S}-r V=0
$$

This is the Black-Sholes partial differential equation and is parabolic type equation as in many financial problems. Furthermore, (10) is called backward parabolic equation since the signs of particular derivatives are the same, namely, they appear on the same side of the equation.

With its extensions and variants, it plays the major role in the option pricing theory. By deriving the partial differential equation for a quantity, such as option price, we hope to be able to find an expression for this value by solving this equation. However a partial equation on its own generally has many solutions. The value of an option should be unique (otherwise, arbitrage possibilities would arise) and so, to pin down the solution, we must also impose boundary conditions. A boundary condition specifies the behavior of the required solution at some part of the solution domain. For the moment we restrict our attention to a European call with value denoted by $C(S ; t)$ with exercise price $E$ and expiry date $T$.

\subsection{Boundary and Final Conditions}

Having derived the Black-Sholes equation for the value of an option, we must next consider final and boundary conditions. But, for the moment we restrict our interest to a European call denoted by $C(S ; t)$, with exercise price $E$ and expiry date $T$. The final condition at time $t=T$ can be derived from the definition of a call option. If at expiration $S>E$ the call option will be worth $S-E$ because the buyer of the option can buy the stock for $E$ and immediately sell it for $S$. If at expiration $S<E$ the option will not be exercised and it will expire worthless. At $t=T$, the value of the option is known for certain to be the payoff

$$
C(S ; t)=\max (S-E, 0)
$$

This is the final condition for our differential equation.

In order to find boundary conditions we consider the value of $C$ when $S=0$ and as $S \rightarrow \infty$ : If $S=0$ then it is easy to see from stochastic differential equation that $\mathrm{d} S=0$, and therefore, $S$ will never change. If at expiry $S=0$ then from (10) the payoff must be 0 . Consequently, when $S=0$ we have

$$
C(S ; t)=0
$$

Now when $S \rightarrow \infty$ it becomes more and more likely the option will be exercised and the payoff will be $S-E$. The exercise price becomes less and less important as $S \rightarrow \infty$, so the value of the option is equivalent to

$$
C(S ; T) \approx S \text { as } S \rightarrow \infty
$$

\subsection{Similarity Solution}

It may occasionally occur that the solution $C(S ; t)$ of a partial differential equation, together with its initial and boundary conditions, depends only on one special combination of the two independent variables. In such cases, the problem can be reduced to an ordinary differential equation in which this combination is the independent 
variable. The solution to this ordinary differential equation is called a similarity solution to the original equation. In [7]; Wilmot et al. have given the similarity solution of the Black-Scholes equation for a European call option (see pages 97-100). The mathematical reasons for the existence of this reduction are subtle and outside of the scope of this paper, although the numerical calculations of the solution given on the Table 1 which, we think, necessary for comparison of our numerical results.

\section{The Mathematical Foundation of Operator Splitting}

In numerous applications in the past revealed that a mixing of the various terms in the equations for the discretization and solver methods made it difficult to solve them together. To overcome this drawback, in 60 's or early 70 's so called the decomposition methods or splitting methods have been introduced [11]. The main idea of these methods is to decouple a complex equation in various simpler equations and to solve the simpler equations with adapted discretization and solver methods. In general, the simpler parts are collected via the initial conditions the results are coupled together. This decoupling procedure allows us to solve a few simpler systems instead of the whole one [11-14].

In this study, we apply operator splitting and the point in operator splitting is the replacement of the original model with one in which appropriately chosen groups of the sub processes, described by the model, take place successively in time. To illustrate the idea, let $S$ denote some normed space and consider the initial value problem

$$
\begin{aligned}
& \frac{\mathrm{d} w(t)}{\mathrm{d} t}=A w(t), t \in(0, T], \\
& w(0)=w_{0},
\end{aligned}
$$

where $w:[0, T] \rightarrow S$ is the unknown function, and $A$ is an operator of type $S \rightarrow S$. Assume that the operator $A$ can be decomposed into a sum of two simpler operators, for example, as $A_{1}$ and $A_{2}$. Then defining the splitting step by $\tau=T / n$, where $n \in \mathbb{N}$, is given, we consider the sequence of initial value problems of the form

$$
\begin{aligned}
& \frac{\mathrm{d} w_{k}^{(1)}(t)}{\mathrm{d} t}=A_{1} w_{k}^{(1)}(t), \quad t \in((k-1) \tau, k \tau] \\
& w_{k}^{(1)}((k-1) \tau)=w_{k-1}^{(2)}((k-1) \tau), \\
& \text { and } \\
& \frac{\mathrm{d} w_{k}^{(2)}(t)}{\mathrm{d} t}=A_{2} w_{k}^{(2)}(t), \quad t \in((k-1) \tau, k \tau], \\
& w_{k}^{(2)}((k-1) \tau)=w_{k}^{(1)}(k \tau),
\end{aligned}
$$

for $k=1,2, \cdots, n$, where $w_{0}^{(2)}(0)=w_{0}$. This procedure is called sequential splitting and can directly be extended to more than two sub operators in a natural way.

Obviously, the alteration of the original problems with the subproblems generally results in some error so called local splitting error. The local splitting error, $E_{n}$, of the sequential operator-splitting method can be given as follows:

$$
\begin{aligned}
E_{n}= & \frac{1}{\tau_{n}}\left(\exp \left(\tau_{n}\left(A_{1}+A_{2}\right)\right)-\exp \left(\tau_{n} A_{2}\right) \exp \left(\tau_{n} A_{1}\right)\right) w_{n}^{1} \\
& +O\left(\tau_{n}^{2}\right)
\end{aligned}
$$

where splitting time step, $\tau_{n}$, is defined by $\tau_{n}=n \cdot t-(n-1) \cdot t$

Now, we extend the method to the Black-Scholes equation given in (10).

\section{The Operator Splitting of the Black-Scholes Equation and Its Numerical Solution}

Splitting methods are important for partial differential equations, because of reducing computational time to solve the equations and accelerating the solver process, see [11]. Based on the splitting launched in Section 3, we have split the Black-Scholes equation given in (10) as follow:

$$
\begin{gathered}
-\frac{\partial V}{\partial t}+\frac{1}{2} \sigma^{2} S^{2} \frac{\partial^{2} V}{\partial S^{2}}+r S \frac{\partial V}{\partial S}=0 \\
-\frac{\partial U}{\partial t}-r U=0
\end{gathered}
$$

Next, we discuss our underlying time- and space-discretization approach for our coupled system of BlackSholes equation given in $17(\mathrm{a}-\mathrm{b})$. Often decoupling methods are applied after discretizing time and space variables. Here, the balance between the time and space discretization methods is important. So, the spatiotemporal schemes can be balanced in implicit-explicit discretization methods. The decoupling in time and space has the advantage of more efficiency and acceleration [11].

\subsection{Finite-Difference Approximation}

Finite-difference methods are one of the resources of obtaining numerical solutions to partial differential equations and linear complimentary problems. They constitute a very powerful and flexible technique, and, if they applied appropriately, competent of producing accurate numerical solutions to all of the model problems arising 
in both the physical and financial sciences.

The underlying idea behind finite-difference methods is to replace the partial derivatives occurring in partial differential equations by approximations based on Taylor's series expansions of functions near the point or points of interest. For example, a partial derivative $\partial u / \partial t$ may be defined to be the limiting difference

$$
\frac{\partial u}{\partial t}(x, t)=\lim _{\delta t \rightarrow 0} \frac{u(x, t+\delta t)-u(x, t)}{\delta t}
$$

If instead of taking the limit $\delta t \rightarrow 0$, we regard $\delta t$ as small but nonzero, hence, we obtain the approximation

$$
\frac{\partial u}{\partial t}(x, t) \approx \frac{u(x, t+\delta t)-u(x, t)}{\delta t}+O(\delta t) \rightarrow 0
$$

This is called a finite-difference approximation or a finite difference of $\partial u / \partial t$ because; it involves small but not infinitesimal, differences of the dependent variable $u$. Furthermore, higher order derivatives can be derived in a similar manner. To continue with the finite difference approximation, we divide the $x$-axis into equally spaced nodes a distance $\delta x=h$ apart, and $t$-axis into equally spaced nodes a distance $\delta t=k$ apart. This divides the $(x, t)$ plane into a mesh, where the mesh points have the form $(i . h, j . k)$. In our case, the grid is made up of the points at asset values $S_{i}=i h$ and times $t=T-j k$ for the convenience.

Balancing of time and spatial discretization here additional balancing is taken into account, and we proposed the Theta methods.

The following theorem, addresses the delicate situation of time and spatial steps and the fact of reducing the theoretical promised order of the scheme:

\subsection{Theta Method}

Detaining our attention to values of $V$ at mesh points, and using appropriate finite- difference for the derivatives in (17a) Theta method reads:

$$
\begin{aligned}
\frac{V_{i}^{j+1}-V_{i}^{j}}{k}= & \frac{1}{2} \sigma^{2} S_{i}^{2}\left(\theta\left(\frac{V_{i-1}^{j}-2 V_{i}^{j}+V_{i+1}^{j}}{h^{2}}\right)\right. \\
& \left.+(1-\theta)\left(\frac{V_{i-1}^{j+1}-2 V_{i}^{j+1}+V_{i+1}^{j+1}}{h^{2}}\right)\right) \\
& +r S_{i}\left(\theta\left(\frac{V_{i+11}^{j}-V_{i-1}^{j}}{2 h}\right)+(1-\theta)\left(\frac{V_{i+1}^{j+1}-V_{i-1}^{j+1}}{2 h}\right)\right)
\end{aligned}
$$

or

$$
\begin{aligned}
& -(1-\theta) A_{i} V_{i-1}^{j+1}+\left(1+(1-\theta) C_{i}\right) V_{i}^{j+1}-(1-\theta) B_{i} V_{i+1}^{j+1} \\
& =\theta A_{i} V_{i-1}^{j}+\left(1-\theta C_{i}\right) V_{i}^{j}+\theta B_{i} V_{i+1}^{j}
\end{aligned}
$$

where

$$
\begin{aligned}
& A_{i}=\left(\frac{1}{2} \sigma^{2} i^{2} k-\frac{1}{2} r i k\right) \\
& B_{i}=\left(\frac{1}{2} \sigma^{2} i^{2} k+\frac{1}{2} r i k\right) \\
& C_{i}=\sigma^{2} i^{2} k
\end{aligned}
$$

Detaining our attention to values of $U$ at mesh points, and using appropriate finite- difference for the derivatives in (17b) Theta method reads:

$$
\frac{U_{i}^{j+1}-U_{i}^{j}}{k}=-r\left(\theta U_{i}^{j}+(1-\theta) U_{i}^{j+1}\right)
$$

or

$$
U_{i}^{j+1}=\frac{(1-r k \theta)}{(1+r k(1-\theta))} U_{i}^{j}
$$

For readers familiar with Theta method or so called weighted average approximation reduces to:

1) The Explicit Finite Difference Method when we take $\theta=1$.

2) The Implicit Finite Difference Method when we take $\theta=0$.

3) The Crank-Nicolson method when we take $\theta=1 / 2$.

Now we will make numerical calculations for each method for the cases 1), 2) and 3) to show the applicability and efficiency of each case for Black-Sholes call option model.

\section{1) Explicit Finite Difference Method}

For Black-Sholes equation, (18), the explicit method is allocated when $\theta=1$, with $\sigma=0.2, r=0.05$ and $E=100$, hence we obtain:

$$
\frac{V_{i}^{j+1}-V_{i}^{j}}{k}=\frac{1}{2} \sigma^{2} S_{i}^{2}\left(\frac{V_{i-1}^{j}-2 V_{i}^{j}+V_{i+1}^{j}}{h^{2}}\right)+r S_{i}\left(\frac{V_{i+1}^{j}-V_{i-1}^{j}}{2 h}\right)
$$

or

$$
V_{i}^{j+1}=A_{i}^{j} V_{i-1}^{j}+\left(1-B_{i}^{j}\right) V_{i}^{j}+C_{i}^{j} V_{i+1}^{j}
$$

where

$$
\begin{aligned}
A_{i}^{j} & =\left(\frac{1}{2} \sigma^{2} i^{2} k-\frac{1}{2} r i k\right) \\
B_{i}^{j} & =\sigma^{2} i^{2} k \\
C_{i}^{j} & =\left(\frac{1}{2} \sigma^{2} i^{2} k+\frac{1}{2} r i k\right)
\end{aligned}
$$

Equation (24) only holds for $i=1, \cdots, M-1$, i.e. for 
interior points, since $V_{-1}^{j}$ and $V_{M+1}^{j}$ are not defined. Thus there are $M-1$ equations for the $M+1$ unknowns, the $V_{i}^{j}$. The remaining two equations come from the two boundary conditions on $i=0$ and $i=M$. These two end points are treated separately.

If we know $V_{i}^{j}$ for all $i$ then Equation (24) tells us $V_{i}^{j+1}$. Since we know $V_{i}^{0}$, the payoff function, we can easily calculate $V_{i}^{1}$.

The second split equation, (21), reduces to

$$
\frac{U_{i}^{j+1}-U_{i}^{j}}{k}=-r U_{i}^{j}
$$

or

$$
U_{i}^{j+1}=(1-r k) U_{i}^{j}
$$

To solve, (27), we take the solution of the first split equation as an initial condition for second split equation, i.e.,

$$
U_{i}^{0}=V_{i}^{1}
$$

and calculate $U_{i}^{1}$ easily, which is the option value of one time step before expiry. Using these values we can work step by step back down the grid as far as we required. Table 2 shows the calculated call option values of Black-Sholes equation. The comparison with exact solutions (see Table 1) shows that obtained results are well-matched with exact ones except for lower strike prices, 110 and 120 .

\section{2) The Implicit Finite Difference Method}

The implicit method for Black-Sholes equation is attained when we take $\theta=0$ in (18). The calculation may be done in similar manner as in case 1) by using basic numerical linear algebra for the linear systems. The comparison with exact solutions (see Table 1) shows that obtained results are well-matched with exact ones except for lower strike prices, 110 and 120 as in the case 1).

\section{3) Crank-Nicolson Method}

The Crank-Nicolson method for Black-Sholes equation is attained when we take $\theta=1 / 2$ in (18). Table 3 shows the calculated call option values of Black-Sholes equation with the Crank-Nicolson method. The comparison with exact solutions (see Table 1) shows that obtained results are well-matched with exact ones except for lower strike prices, 110 and 120 as in the cases 1) and 2 ). The calculation may be done in similar manner as in cases 1) and 2) by using the Crank-Nicolson solver frequently used for solving the linear systems.

\subsection{Weighted Operator Splitting Method (WOSM)}

A more general finite-difference approximation to split
Black-Sholes equation, (17(a)-(b)), than those considered is given by

$$
\begin{aligned}
\frac{V_{i}^{j+1}-V_{i}^{j}}{k} & =\frac{1}{2} w \sigma^{2} S_{i}^{2}\left(\theta\left(\frac{V_{i-1}^{j}-2 V_{i}^{j}+V_{i+1}^{j}}{h^{2}}\right)\right. \\
& \left.+(1-\theta)\left(\frac{V_{i-1}^{j+1}-2 V_{i}^{j+1}+V_{i+1}^{j+1}}{h^{2}}\right)\right) \\
& +w r S_{i}\left(\theta\left(\frac{V_{i+\mid 1}^{j}-V_{i-1}^{j}}{2 h}\right)+(1-\theta)\left(\frac{V_{i+1}^{j+1}-V_{i-1}^{j+1}}{2 h}\right)\right)
\end{aligned}
$$

$$
U_{i}^{j+1}=\frac{(1-r(1-w) k \theta)}{(1+r(1-w) k(1-\theta))} U_{i}^{j}
$$

This approximation may be called as Weighted Operator Splitting Method (WOSM) and we think it is useful for practical consideration for unstable equations. Next we will consider this approximation for the Crank-Nicholson case in the following.

Letting $\theta=1 / 2$ in Equations (28) and (29) where $w \in(0,1)$ is the weighting factor which is determined by trial and error, gives the results shows in Table 4 .

To arbitrate the accuracy of our results given in Tables 2-5, we have calculated numerical values of the explicit (similarity) solution of the Black-Scholes equation for option call problem for call option for $\sigma=0.2$, $r=0.05$ and $E=100$, given in Table 1 .

\section{Conclusions}

In this paper, Black-Scholes equation is solved as a call option problem by variants of splitting method numerically. The comparison of the results obtained by various splitting methods (see Tables 2-5) shows that obtained results are well-matched and the diversity among the numerical values are negligible. This may be considered as the splitting method applied to call option problem is consistent. Our calculations, to some extent, for certain values differ from the values obtained by the similarity solution given in Table 1. We think that the dissimilarity is practically expected and is due to the fundamental nature of the similarity solution. Because, notice that, the similarity solution contains only one parameter, instead of the four parameters $E, \sigma^{2}, T$ and $r$ in the original statement of the problem. The only vital factor controlling the option value is $r / \frac{1}{2} \sigma^{2}$, which is the only dimensionless parameter in the problem. The effect of all other factors is simply brought in by a straightforward arithmetical calculation. On the other hand, the similarity solution technique is rarely successful in solving a com- 
Table 1. Explicit solution for call option for $\sigma=0.2, r=0.05$ and $E=100$.

\begin{tabular}{ccccccccccc}
\hline & $\mathbf{0}$ & $\mathbf{0 . 1 1 1}$ & $\mathbf{0 . 2 2 2}$ & $\mathbf{0 . 3 3 3}$ & $\mathbf{0 . 4 4 4}$ & $\mathbf{0 . 5 5 6}$ & $\mathbf{0 . 6 6 7}$ & $\mathbf{0 . 7 7 8}$ & $\mathbf{0 . 8 8 9}$ & $\mathbf{1}$ \\
\hline $\mathbf{1 1 0}$ & 10 & 10.7514 & 11.7398 & 12.7085 & 13.6288 & 14.511 & 15.3455 & 16.1461 & 16.9173 & 17.663 \\
$\mathbf{1 2 0}$ & 20 & 20.5586 & 21.1794 & 21.8672 & 22.5857 & 23.3198 & 24.0453 & 24.7635 & 25.4718 & 26.169 \\
$\mathbf{1 3 0}$ & 30 & 30.5535 & 31.1096 & 31.6848 & 32.2835 & 32.9058 & 33.534 & 34.1683 & 34.8046 & 35.4403 \\
$\mathbf{1 4 0}$ & 40 & 40.5535 & 41.1042 & 41.6554 & 42.2125 & 42.7832 & 43.3571 & 43.9375 & 44.5226 & 45.1106 \\
$\mathbf{1 5 0}$ & 50 & 50.5535 & 51.1039 & 51.6516 & 52.1984 & 52.7513 & 53.3017 & 53.8552 & 54.4115 & 54.9701 \\
$\mathbf{1 6 0}$ & 60 & 60.5535 & 61.1039 & 61.6513 & 62.196 & 62.7438 & 63.2858 & 63.8278 & 64.3701 & 64.913 \\
$\mathbf{1 7 0}$ & 70 & 70.5535 & 71.1039 & 71.6512 & 72.1956 & 72.7421 & 73.2815 & 73.8191 & 74.3553 & 74.8906 \\
$\mathbf{1 8 0}$ & 80 & 80.5535 & 81.1039 & 81.6512 & 82.1955 & 82.7418 & 83.2804 & 83.8164 & 84.3502 & 84.8821 \\
$\mathbf{1 9 0}$ & 90 & 90.5535 & 91.1039 & 91.6512 & 92.1955 & 92.7417 & 93.2801 & 93.8156 & 94.3485 & 94.8789 \\
$\mathbf{2 0 0}$ & 100 & 100.5535 & 101.1039 & 101.6512 & 102.1955 & 102.7417 & 103.28 & 103.8154 & 104.3479 & 104.8777 \\
\hline
\end{tabular}

Table 2. Call option values output by the explicit code. Stock price ranges from 110 to 200 , time from 0 (expiration) to 1 with $\sigma=0.2, r=0.05$ and $E=100$.

\begin{tabular}{cccccccccccc}
\hline & $\mathbf{0}$ & $\mathbf{0 . 1 1 1}$ & $\mathbf{0 . 2 2 2}$ & $\mathbf{0 . 3 3 3}$ & $\mathbf{0 . 4 4 4}$ & $\mathbf{0 . 5 5 6}$ & $\mathbf{0 . 6 6 7}$ & $\mathbf{0 . 7 7 8}$ & $\mathbf{0 . 8 8 9}$ & $\mathbf{1}$ \\
\hline $\mathbf{1 1 0}$ & 10 & 10.5531 & 11.1032 & 11.6502 & 12.1941 & 12.7399 & 13.2777 & 13.8126 & 14.3444 & 14.8734 \\
$\mathbf{1 2 0}$ & 20 & 20.5529 & 21.1028 & 21.6495 & 22.1933 & 22.7388 & 23.2765 & 23.8111 & 24.3428 & 24.8714 \\
$\mathbf{1 3 0}$ & 30 & 30.5527 & 31.1023 & 31.6489 & 32.1924 & 32.7377 & 33.2752 & 33.8096 & 34.3411 & 34.8695 \\
$\mathbf{1 4 0}$ & 40 & 40.5525 & 41.1019 & 41.6483 & 42.1916 & 42.7367 & 43.2739 & 43.8081 & 44.3394 & 44.8676 \\
$\mathbf{1 5 0}$ & 50 & 50.5523 & 51.1015 & 51.6476 & 52.1907 & 52.7356 & 53.2726 & 53.8066 & 54.3377 & 54.8657 \\
$\mathbf{1 6 0}$ & 60 & 60.5521 & 61.1011 & 61.647 & 62.1899 & 62.7346 & 63.2714 & 63.8052 & 64.336 & 64.8638 \\
$\mathbf{1 7 0}$ & 70 & 70.5519 & 71.1006 & 71.6464 & 72.189 & 72.7335 & 73.2701 & 73.8037 & 74.3343 & 74.8619 \\
$\mathbf{1 8 0}$ & 80 & 80.5517 & 81.1002 & 81.6457 & 82.1882 & 82.7324 & 83.2688 & 83.8022 & 84.3326 & 84.86 \\
$\mathbf{1 9 0}$ & 90 & 90.5514 & 91.0998 & 91.6451 & 92.1873 & 92.7314 & 93.2675 & 93.8007 & 94.3309 & 94.8581 \\
$\mathbf{2 0 0}$ & 100 & 100.5512 & 101.0994 & 101.6445 & 102.1865 & 102.7303 & 103.2663 & 103.7992 & 104.3292 & 104.8562 \\
\hline
\end{tabular}

Table 3. Call option values output by the Crank-Nicolson code. Stock price ranges from 110 to 200, time from 0 (expiration) to 1 with $\sigma=0.2, r=0.05$ and $E=100$.

\begin{tabular}{|c|c|c|c|c|c|c|c|c|c|c|}
\hline & 0 & 0.111 & 0.222 & 0.333 & 0.444 & 0.556 & 0.667 & 0.778 & 0.889 & 1 \\
\hline 110 & 10 & 10.5533 & 11.1036 & 11.6508 & 12.1949 & 12.7409 & 13.2789 & 13.8139 & 14.346 & 14.8751 \\
\hline 120 & 20 & 20.5531 & 21.1032 & 21.6501 & 22.1941 & 22.7398 & 23.2776 & 23.8125 & 24.3443 & 24.8732 \\
\hline 130 & 30 & 30.5529 & 31.1027 & 31.6495 & 32.1932 & 32.7387 & 33.2764 & 33.811 & 34.3426 & 34.8713 \\
\hline 140 & 40 & 40.5527 & 41.1023 & 41.6489 & 42.1924 & 42.7377 & 43.2751 & 43.8095 & 44.3409 & 44.8694 \\
\hline 150 & 50 & 50.5525 & 51.1019 & 51.6482 & 52.1915 & 52.7366 & 53.2738 & 53.808 & 54.3392 & 54.8674 \\
\hline 160 & 60 & 60.5523 & 61.1015 & 61.6476 & 62.1907 & 62.7355 & 63.2725 & 63.8065 & 64.3375 & 64.8655 \\
\hline 170 & 70 & 70.5521 & 71.1011 & 71.647 & 72.1899 & 72.7346 & 73.2713 & 73.8051 & 74.3359 & 74.8638 \\
\hline 180 & 80 & 80.5519 & 81.1007 & 81.6465 & 82.1892 & 82.7337 & 83.2703 & 83.804 & 84.3346 & 84.8623 \\
\hline 190 & 90 & 90.5518 & 91.1004 & 91.646 & 92.1885 & 92.7329 & 93.2693 & 93.8028 & 94.3333 & 94.8608 \\
\hline
\end{tabular}


Table 4. Call option values output by the Weighted Crank-Nicolson code. Stock price ranges from 110 to 200 , time from 0 (expiration) to 1 with $\sigma=0.2, r=0.05$ and $E=100$.

\begin{tabular}{ccccccccccc}
\hline & $\mathbf{0}$ & $\mathbf{0 . 1 1 1}$ & $\mathbf{0 . 2 2 2}$ & $\mathbf{0 . 3 3 3}$ & $\mathbf{0 . 4 4 4}$ & $\mathbf{0 . 5 5 6}$ & $\mathbf{0 . 6 6 7}$ & $\mathbf{0 . 7 7 8}$ & $\mathbf{0 . 8 8 9}$ & $\mathbf{1}$ \\
\hline $\mathbf{1 1 0}$ & 10 & 10.5535 & 11.104 & 11.6514 & 12.1957 & 12.7419 & 13.2803 & 13.8156 & 14.3479 & 14.8774 \\
$\mathbf{1 2 0}$ & 20 & 20.5535 & 21.1039 & 21.6512 & 22.1956 & 22.7417 & 23.28 & 23.8153 & 24.3476 & 24.877 \\
$\mathbf{1 3 0}$ & 30 & 30.5534 & 31.1038 & 31.6511 & 32.1954 & 32.7415 & 33.2797 & 33.815 & 34.3473 & 34.8766 \\
$\mathbf{1 4 0}$ & 40 & 40.5534 & 41.1037 & 41.651 & 42.1952 & 42.7413 & 43.2795 & 43.8147 & 44.3469 & 44.8762 \\
$\mathbf{1 5 0}$ & 50 & 50.5534 & 51.1036 & 51.6509 & 52.1951 & 52.7411 & 53.2792 & 53.8144 & 54.3466 & 54.8758 \\
$\mathbf{1 6 0}$ & 60 & 60.5533 & 61.1035 & 61.6507 & 62.1949 & 62.7409 & 63.279 & 63.8141 & 64.3463 & 64.8755 \\
$\mathbf{1 7 0}$ & 70 & 70.5533 & 71.1035 & 71.6506 & 72.1947 & 72.7407 & 73.2788 & 73.8138 & 74.3459 & 74.8751 \\
$\mathbf{1 8 0}$ & 80 & 80.5532 & 81.1034 & 81.6505 & 82.1946 & 82.7405 & 83.2785 & 83.8136 & 84.3457 & 84.8748 \\
$\mathbf{1 9 0}$ & 90 & 90.5532 & 91.1033 & 91.6504 & 92.1945 & 92.7403 & 93.2783 & 93.8134 & 94.3454 & 94.8745 \\
$\mathbf{2 0 0}$ & 100 & 100.5532 & 101.1033 & 101.6503 & 102.1943 & 102.7402 & 103.2781 & 103.8131 & 104.3451 & 104.8742 \\
\hline
\end{tabular}

Table 5. Call option values output by the implicit code. Stock price ranges from 110 to 200 , time from 0 (expiration) to 1 with $\sigma=0.2, r=0.05$ and $E=100$.

\begin{tabular}{ccccccccccc}
\hline & $\mathbf{0}$ & $\mathbf{0 . 1 1 1}$ & $\mathbf{0 . 2 2 2}$ & $\mathbf{0 . 3 3 3}$ & $\mathbf{0 . 4 4 4}$ & $\mathbf{0 . 5 5 6}$ & $\mathbf{0 . 6 6 7}$ & $\mathbf{0 . 7 7 8}$ & $\mathbf{0 . 8 8 9}$ & $\mathbf{1}$ \\
\hline $\mathbf{1 1 0}$ & 10 & 10.5538 & 11.1045 & 11.6523 & 12.197 & 12.7436 & 13.2823 & 13.818 & 14.3509 & 14.8808 \\
$\mathbf{1 2 0}$ & 20 & 20.554 & 21.105 & 21.6529 & 22.1978 & 22.7446 & 23.2836 & 23.8195 & 24.3526 & 24.8827 \\
$\mathbf{1 3 0}$ & 30 & 30.5542 & 31.1054 & 31.6535 & 32.1987 & 32.7457 & 33.2848 & 33.821 & 34.3543 & 34.8846 \\
$\mathbf{1 4 0}$ & 40 & 40.5544 & 41.1058 & 41.6542 & 42.1995 & 42.7467 & 43.2861 & 43.8225 & 44.356 & 44.8865 \\
$\mathbf{1 5 0}$ & 50 & 50.5546 & 51.1062 & 51.6548 & 52.2004 & 52.7478 & 53.2874 & 53.824 & 54.3577 & 54.8884 \\
$\mathbf{1 6 0}$ & 60 & 60.5548 & 61.1067 & 61.6554 & 62.2012 & 62.7489 & 63.2886 & 63.8255 & 64.3594 & 64.8903 \\
$\mathbf{1 7 0}$ & 70 & 70.5551 & 71.1071 & 71.6561 & 72.2021 & 72.7499 & 73.2899 & 73.827 & 74.361 & 74.8922 \\
$\mathbf{1 8 0}$ & 80 & 80.5553 & 81.1075 & 81.6567 & 82.2029 & 82.751 & 83.2912 & 83.8284 & 84.3627 & 84.8941 \\
$\mathbf{1 9 0}$ & 90 & 90.5555 & 91.1079 & 91.6573 & 92.2037 & 92.752 & 93.2925 & 93.8299 & 94.3644 & 94.896 \\
$\mathbf{2 0 0}$ & 100 & 100.5557 & 101.1083 & 101.658 & 102.2046 & 102.7531 & 103.2937 & 103.8314 & 104.3661 & 104.8979 \\
\hline
\end{tabular}

plete boundary value problems, because it requires such special symmetries in the equation and initial and boundary conditions. Therefore, we can not be confident that the effects we have neglected in making the approximation in both, i.e. splitting method and similarity solution are genuinely unimportant.

\section{References}

[1] R. C. Merton, "Optimum Consumption and Portfolio Rules in a Continuous Time Model," Journal of Economic Theory, Vol. 3, No. 4, 1971, pp. 373-413. doi:10.1016/0022-0531(71)90038-X

[2] R. C. Merton, "Theory of Rational Option Pricing," Bell Journal of Economics and Management Sciences, Vol. 4, No. 1, 1973, pp.141-183. doi:10.2307/3003143

[3] F. Black and M. Scholes, "The Pricing of Options and
Corporate Liabilities," Journal of Political Economy, Vol. 81, No. 3, 1973, pp. 637-654.doi:10.1086/260062

[4] P. Wilmott, S. Howison and J. Dewynne, "The Mathematics of Financial Derivatives," Cambridge University Press, Cambridge, 1997.

[5] P. Wilmott, J. Dewynne and S. Howison, "Option Pricing," Oxford Financial Press, Oxford, 1993.

[6] P. Wilmott, J. Dewynne and S. Howison, "The Mathematics of Financial Derivatives: A Student Introduction," Cambridge University Press, Cambridge, 1997.

[7] P. Wilmott, J. Dewynne and S. Howison, "Option Pricing: Mathematical Models and Computation," Oxford Financial Press, Oxford, 1998.

[8] P. Wilmott, S. Howison and J. Dewynne, "The Mathematics of Financial Derivatives," Cambridge University Press, Cambridge, 1997.

[9] D. Duffy, "Numerical Methods for Instrument Pricing," 
John Wiley \& Sons, Chichester, 2004.

[10] D. J. Duffy, "Financial Instrument Pricing in C++," John Wiley \& Sons, Chichester, 2004.

[11] J. Geiser, "Decomposition Methods for Differential Equations Theory and Applications," Chapman Hall/CRC Press, London, 2009. doi:10.1201/9781439810972

[12] I. Farago and J. Geiser, "Iterative Operator Splitting Methods for Linear Problems," International Journal of Computational Science and Engineering, Vol. 3, No. 4,
2007, pp. 255-263.

[13] P. Csomo's, I. Farago and A. Havasi, "Weighted Sequential Splitting and Their Analysis," Computers \& Mathematics with Applications, Vol. 50, 2005, pp. 1017-1031. doi:10.1016/j.camwa.2005.08.004

[14] J. Geiser, "Linear and Quasi-Linear Iterative Splitting Methods: Theory and Applications," International Mathematical Forum, Vol. 2, No. 49, 2007, pp. 2391-2416. 